\title{
Intelligent dynamic simulation of fed-batch fermentation processes
}

\author{
Esko K. Juuso \\ Control Engineering, Environmental and Chemical Engineering, Faculty of Technology, University of Oulu, Finland, \\ esko.juusodoulu.fi
}

\begin{abstract}
Batch bioprocesses are difficult to model due to strong nonlinearities, dynamic behaviour, lack of complete understanding and unpredictable disturbances. A cell produces more cells, chemical products and heat from chemical substrates. Typical growth characteristics include several phases whose appearances and lengths depend on the type of organisms and the environmental conditions. Large differences exist between different fermentation runs. The simulator developed for fed-batch fermentation processes consists of three interacting dynamic models, each with three phase specific versions. The models predict dissolved oxygen concentration, oxygen transfer rate and concentration of carbon dioxide in the exhaust gas through the whole process, by using only the control variables as inputs. A decision system based on fuzzy logic to provide smooth gradual changes between phases. The detection of the changes between process phases is improved by using the intelligent trend analysis. The dynamic simulator is suitable for an online forecasting tool in connection with the real process. The operation is based on the ideas of model predictive control $(M P C)$ : the previous online measurements on a chosen horizon are used for constructing a starting point and the simulator predicts the operation on a chosen prediction horizon by using the planned control actions. The simulation is started on fairly long time intervals.
\end{abstract}

Keywords: intelligent systems, dynamic simulation, fedbatch fermentation, temporal analysis, prediction

\section{Introduction}

Batch bioprocesses are difficult to model due to strong nonlinearity, dynamic behaviour, lack of complete understanding and unpredictable disturbances from their external environment (Gregersen and Jorgensen, 1999). As every cell in nature has a finite lifetime (Figure 1), a continuous growth of the organisms is needed to maintain the species. The generation time depends on both nutritional and genetic factors. To be able to live, reproduce and make products, a cell must obtain nutrients from its surroundings. The first phase at the beginning of the fermentation is called the lag phase. The second phase is the exponential growth phase. The last phase is called the steady state phase. The secondary metabolic products, such as enzymes, are produced mainly during the steady state phase.

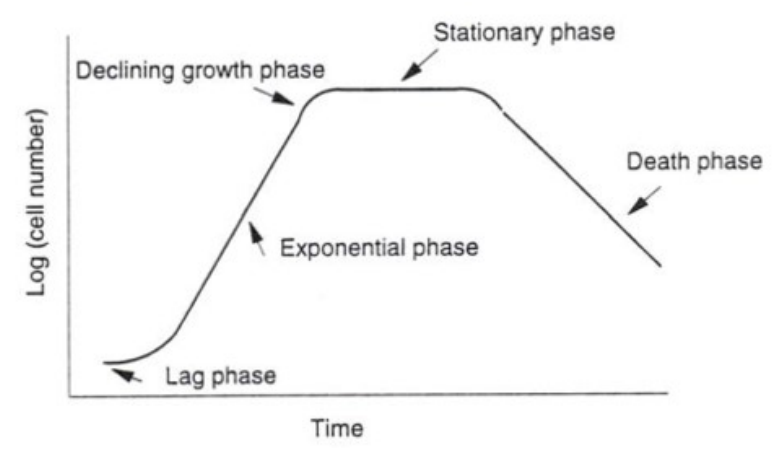

Figure 1. Growth phases in a batch bioprocess (Blanch and Clark, 1997).

In the lag phase, the growth is almost constant caused by many reasons. Since the cells are placed in fresh medium, they might have to adapt to it or adjust the medium before they can begin to use it for growth. Another reason might be that the inoculum is composed partly of dead or inactive cells (Enfors and Häggström, 2000). If a medium consists of several carbon sources, several lag phases might appear. This phenomenon is called diauxic growth. Microorganisms usually use just one substrate at a time and a new lag phase really results when the cells adapt to use the new substrate. (Blanch and Clark, 1997)

The declining of the growth rate begins when a substrate begins to limit. The growth rate slows down until it reaches zero and the stationary phase begins. In the stationary phase, the number of the cells remains practically constant, but the phase is important because many products are only produced during it. The last phase is called the death phase. During the death phase, the cells begin to lyse and the growth rate decreases. (Blanch and Clark, 1997)

In batch reactors, all components, except gaseous substrates such as oxygen, $\mathrm{pH}$-controlling substances and antifoaming agents, are placed in the reactor at the beginning of the fermentation. There is no input nor output flows during the process. In fed-batch processes, nothing is removed from the reactor during the process but one substrate component is added in order to control the reaction rate by its concentration. The process is started as a batch process, and the substrate feed is started when the initial glucose is consumed. The fermentation continues at a cer- 
tain growth rate until some practical limitation inhibits the cell growth (Enfors and Häggström, 2000).

The data sets obtained from the process are in practice distinct sets obtained through different process performances because usually one or more substantial physical parameters, such as dissolved oxygen $(D O)$, temperature or $p H$ are maintained on distinct level (Georgieva et al., 2001). The optimal values of parameters might not be the same for the growth phase and metabolite production phase in secondary metabolite production (Yegneswaran et al., 1991). Large differences exist between different fermentation runs because of the variations in the feeding strategy, the metabolic states of the cells and the amount of oxygen available. Even if the process conditions were kept the same in each fermentation, the micro-organisms would behave differently every time. Detection of fluctuations in operating conditions is essential for making correct actions in time.

The concentration of carbon dioxide $\left(\mathrm{CO}_{2}\right)$ in the exhaust gas is an important variable in a fermentation process since the production of $\mathrm{CO}_{2}$ is correlated to the amount of consumed sugar (Martínez et al., 1999). The variations in the agitation speed can cause changes in the oxygen transfer rate $(O T R)$ and an increase in it can cause an increase in production and yield (Elibol and Ozer, 2000). The $D O$ tension is an important variable in secondary metabolite production and remarkable impacts on production yields can be achieved by affecting this parameter by changes in aeration, agitation system and stirrer speed (Pfefferle et al., 2000). The volumetric mass transfer coefficient, $k_{L} a$, is also an important process variable because it can be used to find the relationship between the $O T R$ and enzyme production (Elibol and Ozer, 2000) and it can be used in the control of the $D O$ tension (Simon and Karim, 2001).

The oxygen requirements of the bacteria differ at different fermentation stages (Yao et al., 2001). By choosing a proper $D O$ tension a product formation can be achieved without wasting the energy source. As the changes are slow, an early forecasting of the process operation is needed. A smoothly operated process is likely to be more productive than one that is subjected to significant disturbances. The aeration supplies oxygen to the process and, at the same time, removes carbon dioxide from microbial cells suspended in the culture broth. The rate of aeration often controls the rates of cell growth and product formation. (Yoshida, 1982)

In fed-batch fermentation, the dynamic simulator has been used online for predicting the process operation in a time window (Saarela et al., 2003a; Juuso, 2005). The results of these tests are used in this research.

This paper analyzes the dynamic simulation model developed for the prediction of the operation in a fed-batch fermentation process. The detection of the phases focuses on the temporal analysis with intelligent trend analysis. The parameters of the prediction models are not changed.

\section{Bioprocess modelling}

A fuzzy predictor presented in (Whitnell et al., 1993) combines three kinds of information: quantitative process inputs, linguistic information and heuristic knowledge from an expert in a beer making process. TakagiSugeno type fuzzy model was used in (Georgieva et al., 2001) on the modelling of a batch biotechnical process, which is strongly influenced by $D O$ concentration as a manipulated input variable. Also black-box and hybrid models have been experimented in the modelling of batch beer production. The research concluded that the extrapolation capability of the model was improved by including mechanical knowledge in the hybrid model. The knowledge based models are useful when only insufficient data from the process can be obtained and they should be thought only as an extension of the ways in which process data can be represented. (Lübbert and Simutis, 1994) Bioprocess parameters have been estimated with neural network models (Simon and Karim, 2001; Thibault et al., 1990; Warne et al., 2004).

Model uncertainties need to be captured for the bioprocess optimization (Liu and Gunawan, 2017). Nonlinear model predictive control $(N M P C)$ and observation of non-measurable states based on an unscented Kalman filter $(U K F)$ were used in (Dewasme et al., 2015).

Temporal reasoning is a very valuable tool for diagnosing and controlling slow processes. Manual process supervision relies heavily on visual monitoring of characteristic shapes of changes in process variables, especially their trends. The fundamental elements are modelled geometrically as triangles to describe local temporal patterns. The elements are defined by the signs of the first and second derivative, respectively (Cheung and Stephanopoulos, 1990).

Linguistic equations introduced in (Juuso and Leiviskä, 1992) have been used in various applications (Juuso, 1999, 2004). Data-driven steady state modelling has been used in the development of linguistic equation $(L E)$ model to represent interactions between measurements:

$$
x_{\text {out }}=f_{\text {out }}\left(-\frac{\sum_{j=1, j \neq \text { out }}^{m} A_{i j} f_{j}^{-1}\left(x_{j}\right)+B_{i}}{A_{\text {iout }}}\right)
$$

where the functions $f_{j}$ and $f_{\text {out }}$ are scaling functions of input variables $x_{j}$ and output $x_{\text {out }}$, respectively. These monotonously increasing, nonlinear functions are generated with generalised norms and moments (Juuso and Lahdelma, 2010). The monotonous increase is ensured with constraint handling. Dynamic structures extend the models to dynamic simulation.

Intelligent trend indices can be calculated from scaled measurements. Triangular episodes are classified with the trend index $I_{j}^{T}(k)$ and the derivative of it, $\Delta I_{j}^{T}(k)$ (Figure 2. Severity of the situations is evaluated by a deviation index which takes into account the scaled values of the measurements (Juuso, 2011). 


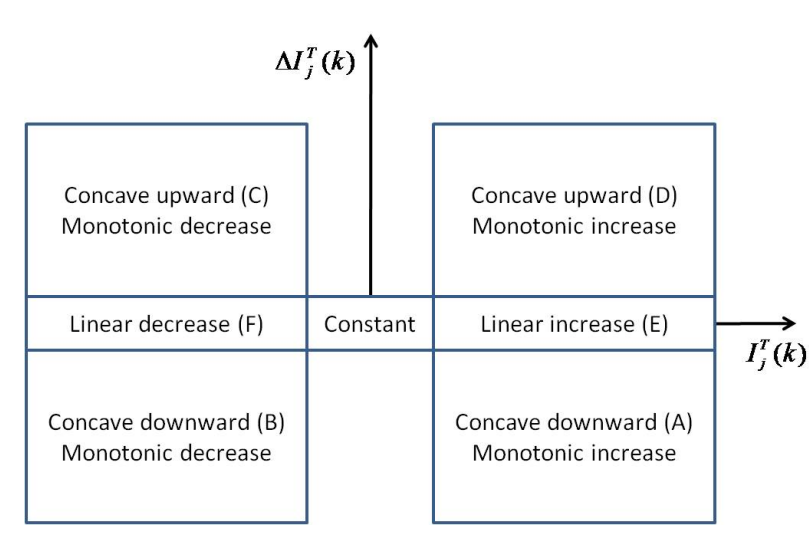

Figure 2. Triangular episodic representations defined by the in$\operatorname{dex} I_{j}^{T}(k)$ and the derivative $\Delta I_{j}^{T}(k)$ (Juuso, 2011).

Linguistic equation method, linear neural network, feedforward neural networks and Takagi-Sugeno fuzzy models created by subtractive clustering appeared to be the best in comparison presented in (Saarela et al., 2003b). The correlations and the relative errors of these models were within acceptable limits and the fuzziness of the models was small. The model surfaces of the models created by these four methods were almost a plane.

\section{Development of dynamic models}

The dynamic models were based on the process data obtained from an industrial fed-batch fermenter. The models were tested using a number of different testing data, which were not included in the training data set. When necessary, the noise in the data was filtered by taking moving averages of the measured values. The variables for each model were chosen mainly based on correlation analysis. Variables that could be used for control were preferred when choosing the input variables of the model. These variables include the mixing rate, aeration, the substrate feed rate etc.

The models have a NARX (Nonlinear AutoRegressive with eXogenous input) structure. A multimodel approach was applied as different growth phases need different models (Figure 3(a)). As the prediction of the future values required three interacting models (Figure 3(b)): each produces the prediction of a different variable, the overall system consists of nine models. Various modelling methodologies have been compared. The compact implementation of the $L E$ models made such a complex structure possible to use. Smooth transitions between the phase models are based on fuzzy decision system (Figure 3(a)).

The controllable variables were preferred as inputs and these include mixing, aeration, feed rate, pressure, temperature and cooling power. The variables used in the models include the concentration of carbon dioxide in the exhaust gas, mixing power, feed rate, oxygen transfer rate, dissolved oxygen concentration, volumetric oxygen transfer coefficient, position of the pressure valve and VVM (vol-

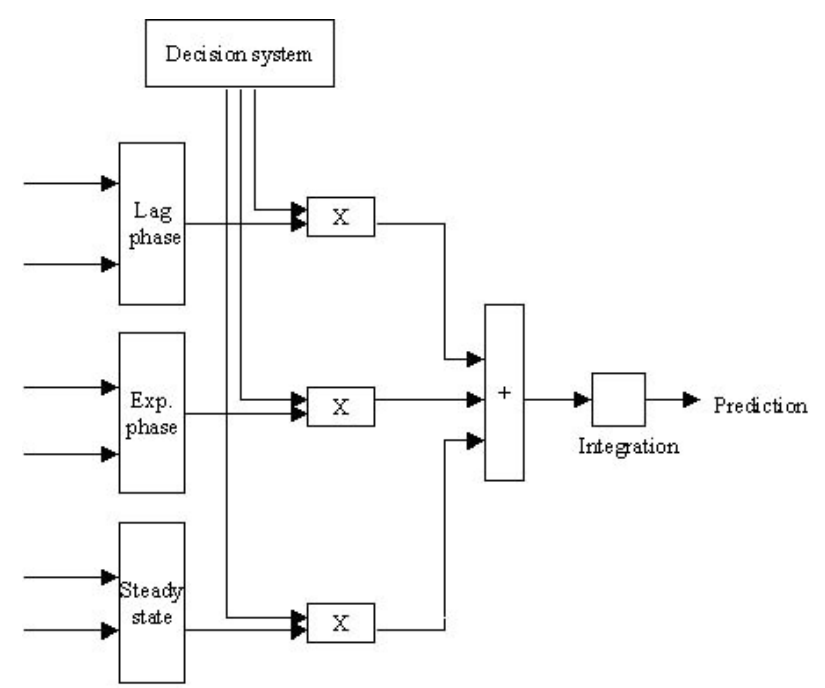

(a) The dynamic model structure of one predicted variable.

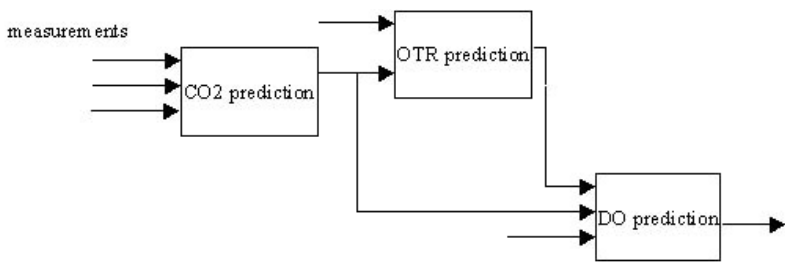

(b) The interactive models.

Figure 3. The overall structure of the model (Saarela et al., 2003a).

umes of air per volume of liquid per minute). The choice of the variables was quite similar to the normal choice in the literature.

Three modelling techniques with several variants were compared including the methods of linguistic equations, neural networks and fuzzy modelling. The steady state modelling of the fermentation variables was not difficult for these intelligent modelling methods: $L E$ models, linear neural network, feedforward neural networks and TakagiSugeno fuzzy models created by subtractive clustering appeared to be the best (Saarela et al., 2003b). However, dynamic simulation turned out to be too demanding for most of these methodologies.

The overall dynamic model shown in Figure 4 contains an additional model for calculating the volumetric mass transfer coefficient, $k_{L} a$.

In the LE models, the definitions of the scaling functions and coefficients $A_{i j}$ from (1) are transferred into the dynamic model. The new prediction is calculated using previous values of the predicted value and the previous values of control variables. Different growth phases can be distinguished from the fermentation process and during these phases different variables affect the output variables. Because of this, three submodels for each predicted variable were created corresponding to each phase in the fermentation process (Figure 3(a)).

The overall model consists of three models and a de- 


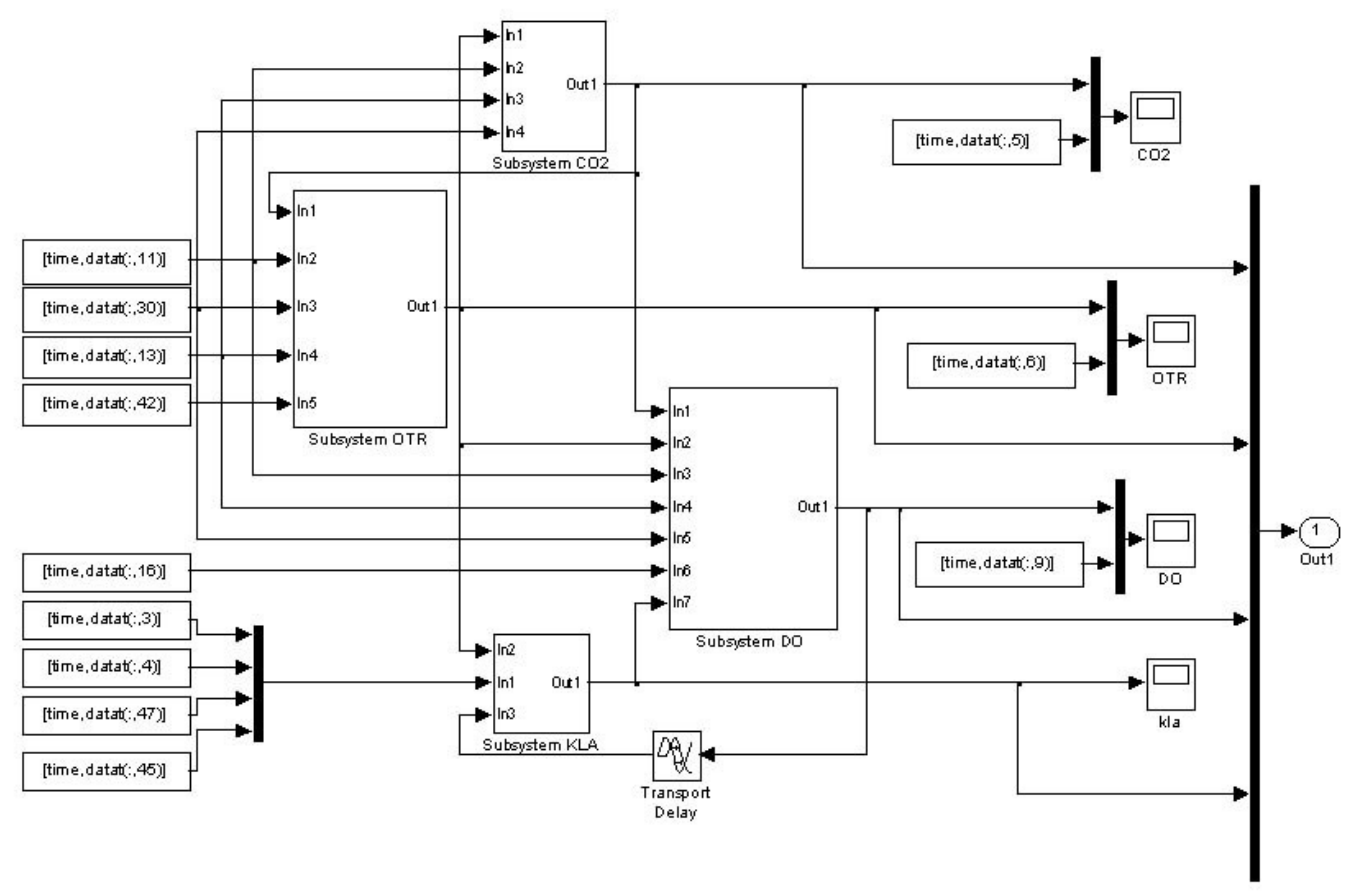

Figure 4. The overall simulation model (Saarela et al., 2003a).

cision system (Figure 3(a). The same structure is used for all the predicted variables. Inputs of the models include measurements from the process, such as mixing power, aeration rate, pressure, and substance concentrations. The inputs to the models of oxygen transfer rate prediction and dissolved oxygen concentration prediction include also predicted values from other models (Figure 3(b)).

Altogether, the overall model contains nine different submodels: three for each predicted variable. The three submodels (lag phase, exponential phase, and steady state) shown in Figure 3(a) form subsystems of the prediction models. The same fuzzy decision system weights the outputs of each of these submodels. In dissolved oxygen model, the coefficients of linguistic equation are $\{0.2,-0.5,0.1,0.1,-0.8\}$.

New predictions are obtained by integrating the calculated changes to the previous value with an ordinary differential equation solver based on an explicit RungeKutta $(4,5)$ formula, the Dormand-Prince pair, with variable step.

The fuzzy decision system chooses the right submodel phase of the process by using the measurements of time, oxygen transfer rate and substrate feed rate. The inference system presented in Figure 5(a) has membership functions for three inputs and one output and a set of eight fuzzy rules for deduction. The system gives a weighting factor from 0 to 1 for each submodel according to which level its results are used. The system was constructed using the Matlab Fuzzy Logic Toolbox. At the beginning of the fermentation for example, the first submodel, the lag phase, is given a weight of one, and the other two submodels have the weight of zero. This means that only the output of the

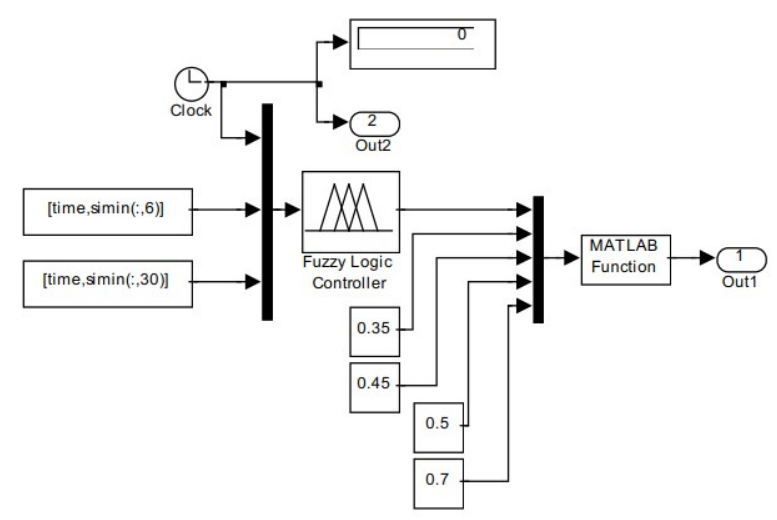

(a) The fuzzy decision system.

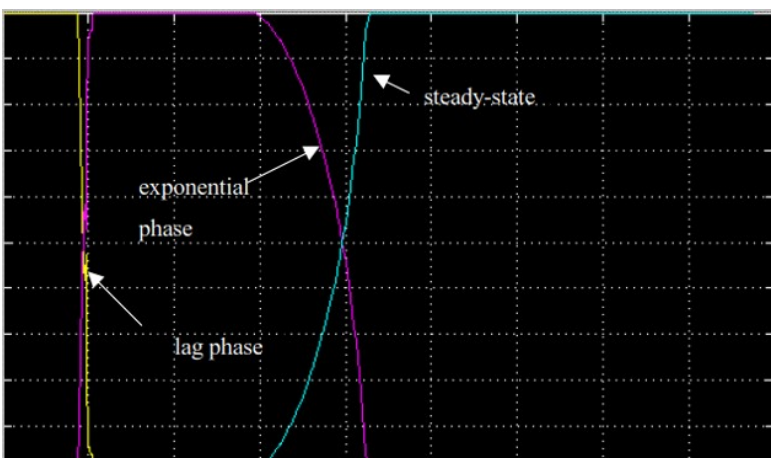

(b) The weights from the decision system.

Figure 5. The decision system for selecting the active phase: $x$ axis represents time and the y-axis weighting factors [0 1], input variables are time, OTR and the glucose feed rate (Saarela et al., 2003a). 
first submodel is used in calculating the prediction. The transition from one phase to another happens smoothly, thus during the transition phase two outputs of the submodels can be used simultaneously (Figure 5(b)).

In the dynamic models, each submodel has been developed separately on the basis of selected training data. The combined model (Figure 4) has been tested with data collected from various fermentation runs. In the simulation tests, the input values were taken from the previously collected data. During the online tests, the prediction system collects the data from the automation system and starts the simulation on chosen time intervals. The prediction results were written back to the data collection system.

\section{Results and discussion}

The models were tested with a set of test data. The fitness of a model can be estimated by examining the correlation, $R$, relative error, fuzziness and the model surfaces. The FuzzEqu program also draws the results of the predictions in the same chart with the test data where they can be compared visually. The fuzziness of the equations should be close to zero. It shows how well the equation represents the data (Juuso, 1999).

\subsection{Steady-state simulation}

First, steady-state models for all three variables were made by the linguistic equations approach. Correlations of the dissolved oxygen models for different testing data were between [0.88-0.98] and the relative errors between [0.030.18]. For models of oxygen transfer rate the correlations were between [0.72-0.99] and the relative errors between [0.02-0.33]. Similar results were obtained with all the static models used in the simulation model. The first part of the process was the most difficult to model, largely due to differences between fermentations. However, at the beginning of the process the concentration of the dissolved oxygen is usually quite high and its predicted value is not so critical.

An example of data-driven modelling results for the prediction of the dissolved oxygen is presented in Figure 6 . The new measure, fuzziness, is used for detecting areas where the models should be considerably different. Fuzziness can also be considered as an additional unknown variable. In this case, the fuzziness is very low.

\subsection{Dynamic simulation}

Dynamic modelling and simulation was performed in Matlab Simulink. Figure 5(b) presents the weights of the submodels obtained from the fuzzy decision system. The change from one phase to another is quite fast. The estimation of the dissolved oxygen concentration is presented in Figure 7(a). In this model, the estimations of the oxygen transfer rate and the concentration of carbon dioxide are used as inputs. The estimation of the oxygen transfer rate can be seen in the Figure 7(b). The estimate for the carbon dioxide concentration is used as an input of the
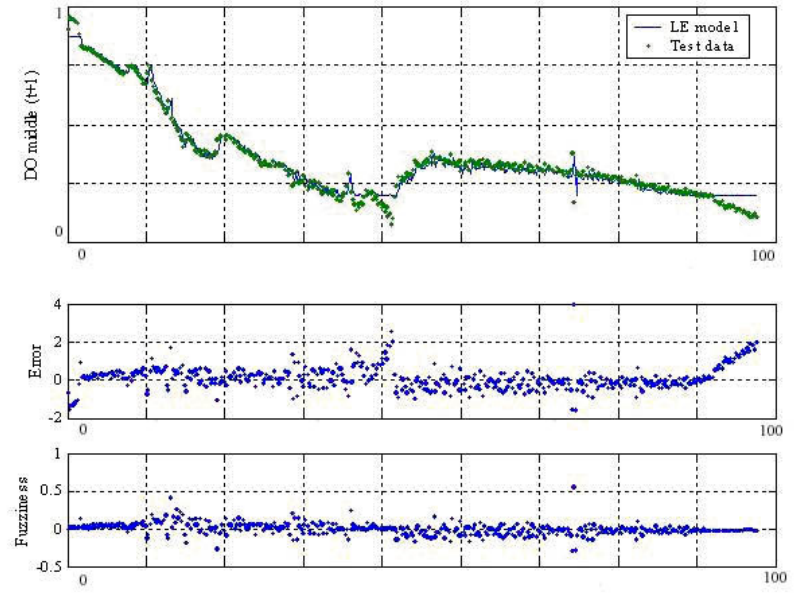

Figure 6. Results from the testing of steady-state fermentation models. Time from 0 to 100 is shown on the $\mathrm{x}$-axis and the values of dissolved oxygen concentration, error and fuzziness on the y-axis (Saarela et al., 2003a).

model. The correlations and relative errors of these results are shown in the figures. With the exception of a few fermentations that largely differed from the others, the results were similar for other test data. The estimation was easier for the oxygen transfer rate and the carbon dioxide concentration than for dissolved oxygen concentration.

A multimodel approach was applied as different growth phases need different models. As the prediction of the future values required three interacting models, which each produces the prediction for a different variable, the overall system consists of nine models. The compact implementation of the $L E$ models made such a complex structure possible to use. Smooth transitions between the phase models are based on fuzzy logic.

The important factors in the success of the modelling were the choice of the input variables, the choice of the model type and structure, and the choice of training data. The training data should be sufficiently large so that it can represent different fermentations. The results of the modelling can improve with the number of data runs employed for training (de Azevedo et al., 1997). Large differences exist between different fermentation runs because of the variations in the feeding strategy, the metabolic state of the cells and the amount of oxygen available. Even if the process conditions were the kept same in each fermentation, the micro-organisms would behave differently every time.

The choice of the input variables was difficult. Different variables affect the output variables in the different phases of the process. All the influence of the variables could not be examined because the data was obtained from an industrial fermenter and a part of the variables were controlled to remain constant. The data based modelling methods require changes in the data to be able to model it.

The dynamic simulator operates accurately throughout the fermentation even for more than 40 hours as a real simulation, i.e. the simulator uses in each time step only 
the previous simulated value and the values of the variables which control the process, according to the dynamic model. Differences between the calculated and measured are reasonable and provide a good basis for detecting fluctuations in operating conditions.

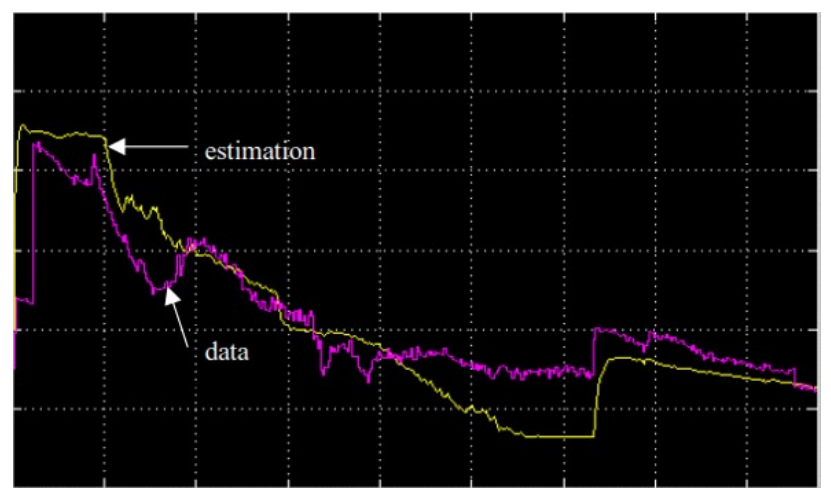

(a) Prediction of dissolved oxygen $(D O)$ concentration: time is on the $\mathrm{x}$-axis and the dissolved oxygen concentrations on the $\mathrm{y}$-axis.

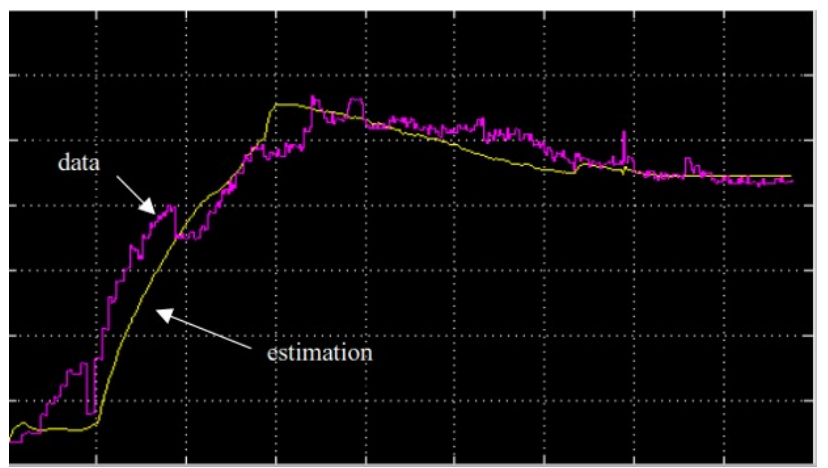

(b) Prediction of oxygen transfer rate $(O T R)$ : time is on the $\mathrm{x}$-axis and the oxygen transfer rates on the $y$-axis.

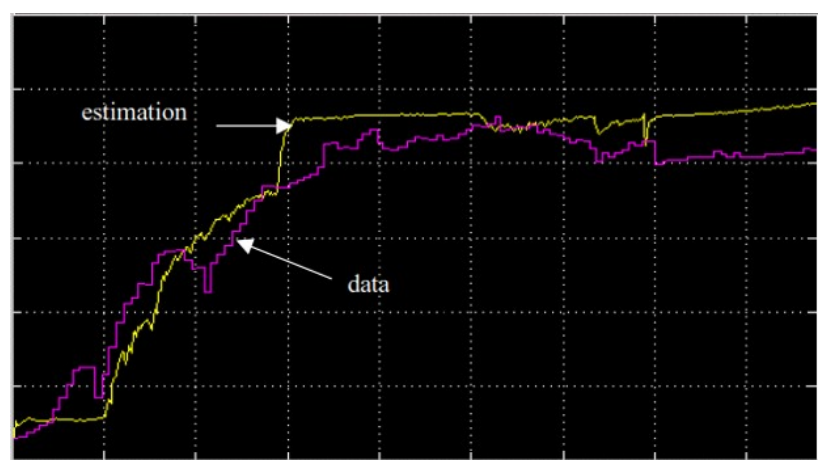

(c) Prediction of carbon dioxide $\left(\mathrm{CO}_{2}\right)$ : time is on the $\mathrm{x}$-axis and the oxygen transfer rates on the y-axis.

Figure 7. Simulation results of a fermentation run (Saarela et al., 2003a)

The simulator is aimed primarily on the detection of changes and fluctuations for the process control. In the estimation, the starting time of the growth phase was predefined. However, the test results reveal a diauxic growth: the first growth starts earlier as can be seen in all predictions which is seen in decreasing $D O$ (Figure 7(a) and increasing $\mathrm{OTR}$ and $\mathrm{CO}_{2}$ (Figures 7(b) and 7(c)). Updating the parameter of the scaling functions with newer methodologies (Juuso and Lahdelma, 2010) would be beneficial.

The drop of $D O$ during the first phase introduces a new lag phase of the second growth phase, which starts later, proceeds slower than the first growth phase and finally slows down gradually to the stationary phase. Two models with different parameter tuning are are required for the growth phase. The stationary phase has two stages: the first part fairly constant $O T R$ but then the death phase is partly activated. Aeration stabilizes the $O T R$ on a new constant level. The estimation errors seen in Figure 7 are at least partly caused by the errors in the fuzzy decision system. Clearly time, OTR and the glucose feed rate are not sufficient for defining the start of the growth phase and the diauxic growth needs to be taken into account.

\subsection{Detection of operating conditions}

The simulator can be used as an online forecasting tool in connection with the real process. The simulator is started on chosen time intervals: the previous online measurements on a chosen horizon are used for constructing a starting point and the simulator predicts the operation on a chosen prediction horizon by using the planned control actions. In the online tests, the prediction horizon has been one hour and the time interval between predictions six minutes. The model predictive control can be considered as a new option since the simulator is very compact. Actually, generating a good starting point for simulation calculations was more demanding than the prediction part. This operates well in the stationary phase. The simulator is started on chosen time intervals and it operates accurately throughout the fermentation even for more than 40 hours (Juuso, 2005).

The intelligent trend analysis improves the detection of phase changes (Figure 1) by using triangular episodes shown in Figure 2: the start of the growth phase is seen as a concave upward monotonic increase (Episode D) which continues as a linear increase (Episode E). The slowdown is detected as Episode A. The activation of the dead phase is seen with Episodes B, F and C. The analysis, which proceeds with time, is adapted by short and long time windows to the speed of the process. Differencies of the fermentation runs are essential in the analysis.

\section{Conclusions}

The simulator can be used as an online forecasting tool in connection with the real process in the stationary phase. The operation is based on the ideas of model predictive control (MPC). In this case, the simulation is started on fairly long time intervals. The previous online measurements on a chosen horizon are used for constructing a starting point and the simulator predicts the operation on a chosen prediction horizon by using the planned control actions. Intelligent trend analysis provides efficient tools for the early detection of the changes in operation phases and situations. The solution adapts to differences in fermentation runs. 


\section{References}

H. W. Blanch and D. S. Clark. Biochemical Engineering. CRC Press, 1997. 702 pp.

J. T.-Y. Cheung and G. Stephanopoulos. Representation of process trends - part I. A formal representation framework. Computers \& Chemical Engineering, 14(4/5):495-510, 1990.

S. Feyo de Azevedo, P. Dahm, and R. R. Oliveira. Hybrid modelling of biochemical processes: A comparison with the conventional approach. Computers and Chemical Engineering, 21(Suppl.):S751-756, 1997.

L. Dewasme, S. Fernandes, Z. Amribt, L.O. Santos, Ph. Bogaerts, and A. Vande Wouwer. State estimation and predictive control of fed-batch cultures of hybridoma cells. Journal of Process Control, 30:50 - 57, 2015. doi:10.1016/j.jprocont.2014.12.006.

M. Elibol and D. Ozer. Influence of oxygen transfer on lipase production by rhizopus arrhizus. Process Biochemistry, 36: 325-329, 2000.

S.-O. Enfors and L. Häggström. Bioprocess Technology Fundamentals and Applications. Royal Institute of Technology, Stockholm, 2000. 356 pp.

O. Georgieva, M. Wagenknecht, and R. Hampel. Takagi-sugeno fuzzy model development of batch biotechnological process. International Journal of Approximate Reasoning, 26:233250, 2001.

L. Gregersen and S. B. Jorgensen. Supervision of fed-batch fermentation. Chemical Engineering Journal, 75:69-76, 1999.

E. Juuso and S. Lahdelma. Intelligent scaling of features in fault diagnosis. In 7th International Conference on Condition Monitoring and Machinery Failure Prevention Technologies, CM 2010 - MFPT 2010, 22-24 June 2010, Stratfordupon-Avon, $U K$, volume 2, pages 1358-1372, 2010. URL Www. scopus. com.

E. K. Juuso. Fuzzy control in process industry: The linguistic equation approach. In H. B. Verbruggen, H.-J. Zimmermann, and R. Babuška, editors, Fuzzy Algorithms for Control, International Series in Intelligent Technologies, volume 14 of International Series in Intelligent Technologies, pages 243300. Kluwer, Boston, 1999. doi:10.1007/978-94-011-44056_10.

E. K. Juuso. Integration of intelligent systems in development of smart adaptive systems. International Journal of Approximate Reasoning, 35(3):307-337, 2004. doi:10.1016/j.ijar.2003.08.008.

E. K. Juuso. Dynamic simulation of a fed-batch enzyme fermentation process. In Proceedings of SIMS 2005, 46th Conference on Simulation and Modeling, October 13-14, 2005, Trondheim, Norway, pages 117-124. Tapir Academic Press, Trondheim, 2005. ISBN 82-519-2093-0.

E. K. Juuso. Intelligent trend indices in detecting changes of operating conditions. In 2011 UKSim 13th International Conference on Modelling and Simulation, pages 162-167. IEEE Computer Society, 2011. doi:10.1109/UKSIM.2011.39.
E. K. Juuso and K. Leiviskä. Adaptive expert systems for metallurgical processes. In S.-L. Jämsä-Jounela and A. J. Niemi, editors, Expert Systems in Mineral and Metal Processing, IFAC Workshop, Espoo, Finland, August 26-28, 1991, IFAC Workshop Series, 1992, Number 2, pages 119-124, Oxford, UK, 1992. Pergamon.

Y. Liu and R. Gunawan. Bioprocess optimization under uncertainty using ensemble modeling. Journal of Biotechnology, 244:34 - 44, 2017. doi:10.1016/j.jbiotec.2017.01.013.

A. Lübbert and R. Simutis. Using measurement data in bioprocess modelling and control. Trends in Biotechnology, 12(8): 304 - 311, 1994. doi:10.1016/0167-7799(94)90047-7.

G. Martínez, A. López, A. Esnoz, P. Vírseda, and J. Ibarrola. A new fuzzy control system for white wine fermentation. Food Control, 10:175-180, 1999.

C. Pfefferle, U. Theobald, H. Gürtler, and H.-P. Fiedler. Improved secondary metabolite production in the genus streptosporangium by optimisation of the fermentation condition. Journal of Biotechnology, 80:135-142, 2000.

U. Saarela, K. Leiviskä, and E. Juuso. Modelling of a fed-batch fermentation process. Report A 21, June 2003. Control Engineering Laboratory, University of Oulu, Oulu, 2003a.

U. Saarela, K. Leiviskä, E. Juuso, and A. Kosola. Modelling of a fed-batch enzyme fermentation process. In IFAC International Conference on Intelligent Control Systems and Signal Processing. Faro, Portugal, April 8-11, 2003. IFAC, 2003b.

L. Simon and M. Nazmul Karim. Identification and control of dissolved oxygen in hybridoma cell culture in a shear sensitive environment. Biotechnology Progress, 17:634-642, 2001.

J. Thibault, V. V. Breusegem, and A. Cheruy. On-line prediction of fermentation variables using neural networks. Biotechnology and Bioengineering, 36(12):1041-1048, 1990.

K. Warne, G. Prasad, S. Rezvani, and L. Maguire. Statistical and computational intelligence techniques for inferential model development: a comparative evaluation and a novel proposition for fusion. Engineering Applications of Artificial Intelligence, 17:871-930, 2004.

G. P. Whitnell, V. J. Davidson, R. B. Brown, and G. L. Hayward. Fuzzy predictor for fermentation time in a commercial brewery. Computers Chemical Engineering, 17(10):1025-1029, 1993.

H. M. Yao, Y. C. Tian, M. O. Tadé, and H. M. Ang. Variations and modelling of oxygen demand in amino acid production. Chemical Engineering and Processing, 40:401-409, 2001.

P. K. Yegneswaran, M. R. Gray, and B. G. Thompson. Effect of dissolved oxygen control on growth and antibiotic production in streptomyces clavuligerus fermentation. Biotechnology Progress, 10:246-250, 1991.

F. Yoshida. Chapter 1 - aeration and mixing in fermentation. volume 5 of Annual Reports on Fermentation Processes, pages 1 - 34. Elsevier, 1982. doi:10.1016/B978-012-040305-9.50005-4. 